\title{
Research on Coupling of Low Speed Straight-Flow Wind Tunnel Based on Ansys Workbench
}

\author{
Zhang Yifei", Qiu Rongkai, Shen Jiang \\ Equipment Design and Instrument Research Institute, China Aerodynamics Research and Development Center, Mianyang, China
}

\section{Email address:}

zhangyifei@cardc.cn (Zhang Yifei), huangqrk@163.com (Qiu Rongkai), sj190378349@163.com (Shen Jiang)

${ }^{*}$ Corresponding author

\section{To cite this article:}

Zhang Yifei, Qiu Rongkai, Shen Jiang. Research on Coupling of Low Speed Straight-Flow Wind Tunnel Based on Ansys Workbench. Science Discovery. Vol. 5, No. 5, 2017, pp. 380-384. doi: 10.11648/j.sd.20170505.23

Received: July 13, 2017; Accepted: September 4, 2017; Published: September 14, 2017

\begin{abstract}
Introduction of research on coupling of low speed straight-flow wind tunnel based on ANSYS Workbench. Wind tunnel is a most important test method of aerodynamics research, in which the air velocity field and pressure field determine the test research level. A computational fluid dynamics simulation based on ANSYS Fluent is conducted on wind tunnel, together with a coupling analysis of the fluid and wind tunnel structure, which helps us make further research on wind tunnel's air field and structure. This work contributes to better operating of test research and wind tunnel optimization design.
\end{abstract}

Keywords: Straight-Flow Wind Tunnel, Low Speed Wind Tunnel, Fluid-Solid Coupling, ANSYS Workbench

\section{基于Ansys Workbench的直流式低速风洞流固耦合的研究}

张逸飞*, 邱荣凯, 申江

设备设计及仪器研究所, 中国空气动力研究与发展中心, 绵阳市, 中国

邮箱

zhangyifei@cardc.cn（张逸飞）, huangqrk@163.com（邱荣凯）, sj190378349@163.com（申江）

摘要: 介绍了基于Ansys Workbench的直流式风洞流固耦合分析。直流式风洞是研究空气动力学试验的重要手段, 而风 洞中的气流速度场、压力场等的品质决定了该试验研究手段的水平。结合ICEM CFD对风洞进行计算流体仿真, 并开 展流体和洞体结构耦合仿真分析, 研究该风洞的流场和结构, 对于更好地利用风洞开展试验研究, 以及优化风洞设计 有着非常重要的意义。

关键词：直流式风洞，低速风洞，流固耦合，数值计算

\section{1. 引言}

空气动力学研究有三大重要手段, 一是理论研究, 二 是实验研究, 三是数值计算。三种重要手段相辅相成, 共 同推动了当今空气动力学事业的蓬勃发展。风洞是进行空 气动力学实验研究的基础设备。风洞设计一般是根据理论 研究和工程经验, 而数值计算仿真具有信息量大、成本低、
响应快、并行性高等优点[1], 可以辅助风洞空气动力学性 能及结构设计。

目前流固耦合问题按照耦合机理可以分为两类, 第一 类是流体和固体部分或全部重叠, 其本构方程需要针对具 体的物理现象来建立, 而耦合效应需要通过描述问题的微 分方程; 第二类是流体和固体耦合发生在两相介质的界面 上, 其耦合方程由耦合面上的平衡及协调关系来建立。风 
洞内部气流与洞体结构的耦合问题就是属于第二类[2]。通 过流固耦合仿真, 深入认识风洞运行过程中高速气流对风 洞结构的影响, 发现并改造不良结构, 提升风洞性能, 提 升空气动力学的研究水平。

目前空气动力学数值计算研究手段已经非常成熟, 有 PHONENICS、STAR-CD、CFX、FLUENT等商业软件[3]。 本文中流固耦合仿真基于ANSYS Workbench14.5, 流体分 析采用了ANSYS集成的FLUENT模块, 结构分析采用了 ANSYS集成的STATIC STRUCTURE模块, 网格划分采用 了基于集成模块 CFX的MESH智能划分。由于ANSYS接口 广泛, 直流风洞的结构设计采用的是NX8.5, 并在ANSYS Design Modeler中优化结构模型[4]。

\section{2. 直流式风洞结构}

直流式低速风洞的结构特点与回流式低速风洞互相 对应。如图 1 所示为中国空气动力研究与发展中心 (CARDC) 的一座回流式低速风洞, 其试验段截面尺寸 为 $1.8 \mathrm{~m} \times 1.4 \mathrm{~m}$ 。回流式风洞具有封闭的气流通道, 能够获 得更好的流场品质和更高的气流速度, 但结构更为复杂、 占地面积更大, 成本更高。而直流式风洞有着相对简单的 结构, 如图2所示, 只有一个单方向通道, 进气口和出气 口连接大气, 生产成本较低, 占地面积较小 [5]。相比较而 言, 直流式风洞一般要比同等级同类型的回流式风洞在洞 体结构上少了一到两个扩散段、四个拐角段、两个回流段 以及相应的基座结构等。

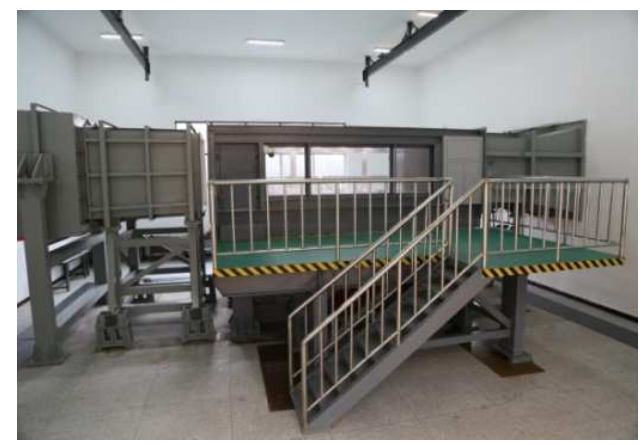

(a) CARDC $1.8 \mathrm{~m} \times 1.4 \mathrm{~m}$ 低速风洞试验段

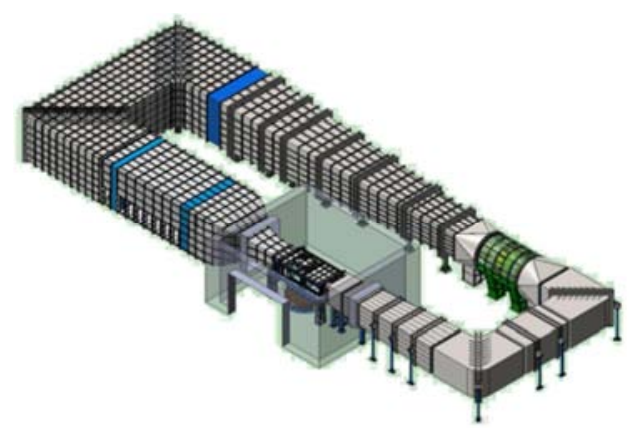

(b) CARDC $1.8 \mathrm{~m} \times 1.4 \mathrm{~m}$ 低速风洞轮廓
在实际工程实践中, 风洞根据模拟高空风速的试验需 求一般建造为低速风洞、亚声速风洞、跨声速风洞、高速 风洞、高超声速风洞。其中, 低速风洞的风速能力一般为 0.3 马赫以下, 亚声速风洞的风速能力一般为 $0.3 \sim 0.8$ 马赫, 跨声速风洞的风速能力一般为 $0.8 \sim 1.4$ 马赫, 高速风洞的风 速能力一般为 $1.4 \sim 4.5$ 马赫, 高超声速风洞的风速能力一般 为5马赫以上。而直流式风洞由于结构简单, 两侧出入风 口直接接连大气, 一般为低速常规风洞。

对于某些有着特殊试验需求的风洞而言, 比如声学风 洞, 结冰风洞等, 这类有着特殊的模拟高空飞行特别大气 环境的风洞, 则无法通过直流式风洞来实现。但正是由于 直流式风洞对于模拟一般大气环境拥有结构简单、成本低 廉、建造周期短、适用范围广等优点, 各个规模尺寸的这 类风洞被广泛大量建造, 为风洞试验及研究提供了重要的 基础和巨大的助力。

\section{1. 风洞结构}

图2(a)介绍了ANSYS Design Modeler中某直流式风洞 的主体结构模型。该直流式风洞总长约27米, 从右至左主 要包括唇口段、稳定段、收缩段、试验段、圆变方段、第 一扩散段、风扇段、第二扩散段等。

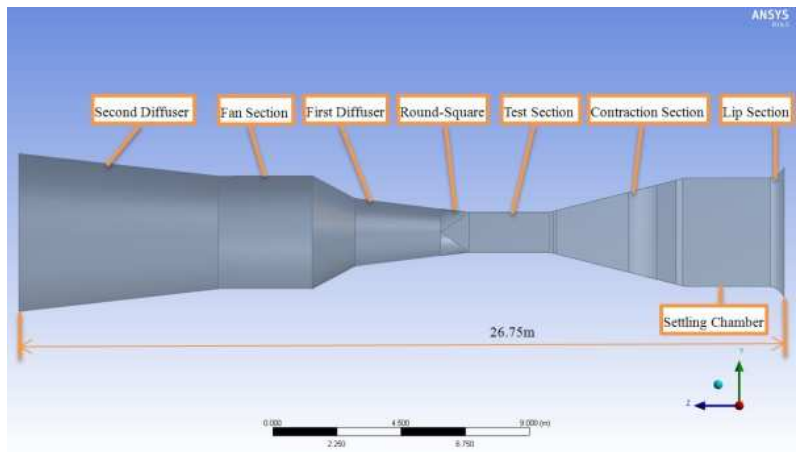

(a) CARDC某直流式风洞轮廓

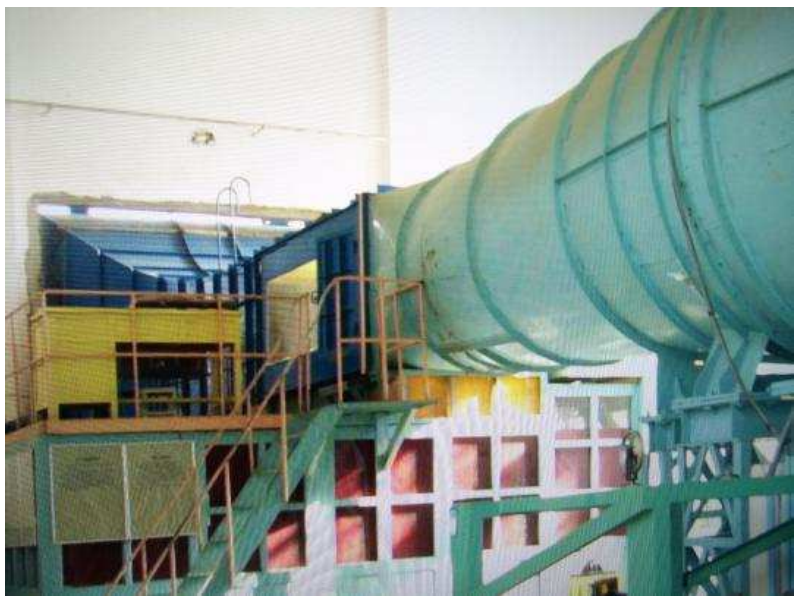

(b) CARDC某直流式风洞局部

图1 CARDC 1.8m×1.4m回流式风洞。 


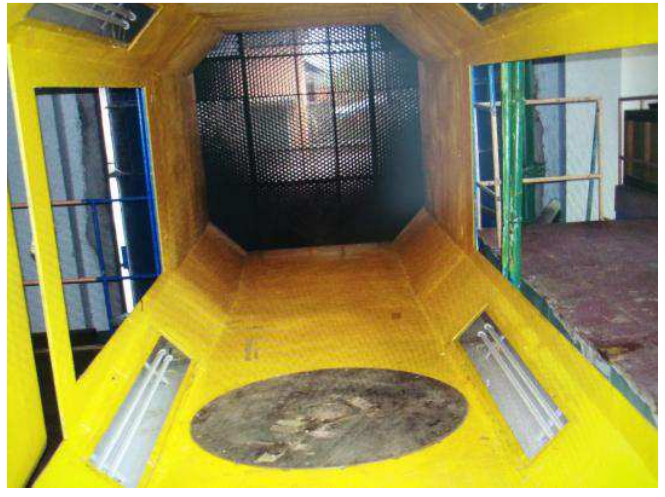

(c) CARDC某直流式风洞试验段内部

图2 CARDC某直流式风洞结构示意图。

从结构设计上来讲, 唇口段是整个直流式风洞的入口, 其上安装有阻尼网, 如图2(c)所示, 从试验段内部向通道 一侧可以看到, 用以过滤随风而至的杂物和鸟类等。从气 动声学上来讲, 该段作为稳定段延伸的弧形扩张结构, 可 以更好的实现风洞气流的平稳进出, 也避免了不连续结构 带来的紊流和噪声; 稳定段是一段长直结构, 主要功能是 让进入风洞的气流逐渐平稳; 收缩段为梯形收缩结构, 风 洞截面尺寸在此急剧缩小, 主要功能是让气流提速, 以达 到模拟飞行试验的需求; 试验段是风洞的核心部段, 是试 验物品如飞机模型、导弹模型、汽车模型等的测试地点。 如图2(c)所示, 试验段一般在壁面开有人孔, 供工作人员 进出维护调试, 内部安装有各种测量调校设备, 用以测量 记录模型在风洞试验过程中各个阶段各个部位受到的压 力、产生的变形等。根据这些试验数据和分析, 可以看到 初步设计中的各种气动、结构、声学等方面的问题, 从而 进一步优化设计; 圆变方段, 实现风洞主体结构从方形试 验段向圆形扩散段的过渡; 风扇段, 作为风洞的动力源, 决定了试验段能达到的风速等级; 第一扩散段、第二扩散 段, 逐步扩大风洞截面尺寸, 降低风速。

\section{2. 结构简化}

考虑到风洞结构的复杂性和流固耦合特点, 风洞模型 中去掉了风洞的阻尼网、内置风扇、人孔等局部细节。此 外, 由于洞体结构的支座约束由于刚度远大于风洞洞体刚 度, 因此在后续数值计算中不考虑支座模型, 改为对应固 定点约束 [6]。

\section{ANSYS FLUENT仿真}

基于ANSYS FLUENT的流体仿真是利用FLUENT模 块对风洞内部气流进行边界设定、基于ICEM MESH网格 划分、求解及后处理的过程。

\section{1. 网格划分}

在三维实体网格中, 六面体网格具有求解精度高、抗 变形能力强、所需网格数量少等优点, 因而在网格划分中 得到了优先的考虑[7]。

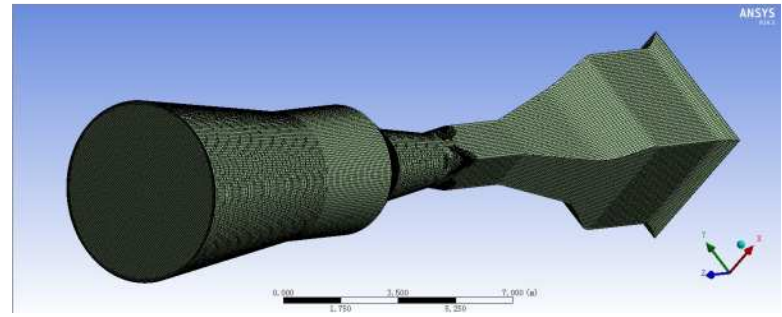

(a) 风洞气流网格

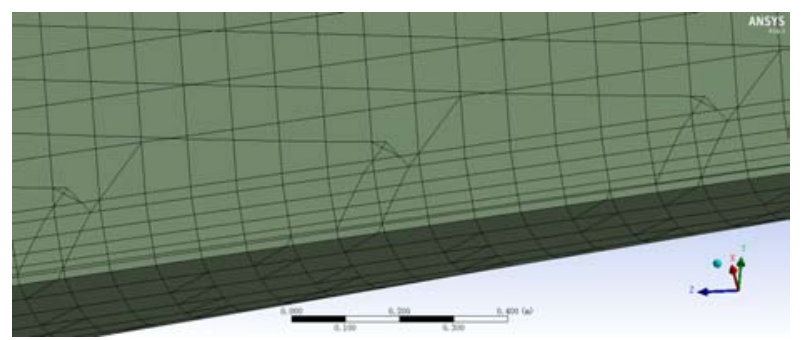

(b) 六面体占优网格局部

图3 风洞内部气流网格划分。

图3为风洞内部气流网格。常用的六面体网格自动划 分方法有映射法、扫描法、铺路/铺层法、基于栅格法等。 该直流式低速风洞内部气流网格划分基于ANSYS MESH, 集成了ICEM网格划分的功能, 主体采用六面体网格智能 划分, 同时对内部复杂结构进行复合网格智能优化, 这样 有利于提高网格质量和划分效率 $[8]$ 。

通过这种划分方式，由于六面体网格更能贴合流场方 向, 在流场边界层的地方比四面体网格离散误差要小, 流 体仿真结果能更加贴近真实试验情况。网格节点数约 85.6 万, 网格总数 87.8 万。

\section{2. 边界条件}

该直流式风洞唇口一侧为入口, 入口气流速度为 $6 \mathrm{~m} / \mathrm{s}$ 。 第二扩散段一侧为出口, 出口气流静压为一个标准大气压。 仿真模型标准可以直接采用标准k-E模型, 考虑到该 直流式风洞为低速风洞, 设置湍流度为 0 。

风洞内壁即内部气流模型外壁采用无滑移速度边界 条件。

\section{3. 仿真结果}

图4所示为风洞内部气流在特定工作条件下的速度场 分布。根据仿真结果图4 (b) 可以得知, 在入口风速 $6 \mathrm{~m} / \mathrm{s}$ 、 出口气流静压为一个标准大气压的工作条件下, 该直流式 低速风洞运行时试验段最大风速为 $70 \mathrm{~m} / \mathrm{s}$ 。考虑到风洞收 缩段曲线经过优化修正, 风洞环境对测量和模型简化的影 响, 仿真结果与直流式低速风洞试验测得试验段最大风速 $65 \mathrm{~m} / \mathrm{s}$ 吻合良好[9]。由此也可以验证该模型算法的合理性。 


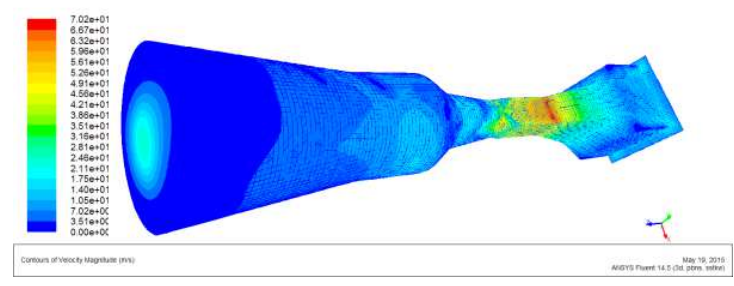

（a）去除表面边界速度场

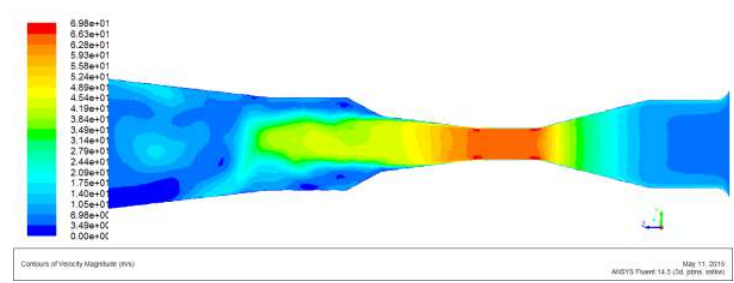

(b) 过轴心水平面速度场

图4 风洞内部气流速度场。

图5为风洞在试验段最大风速运行条件下，内部气流 产生的压力场。可以看到, 此时风洞试验段气流压力最小, 而入口处气流压力最大, 同时根据上述速度场分布图 4 和 平时实际试验结果得知, 在该工作条件下试验段风速最大 而入口处风速最小。这与流体理论中的伯努利原理吻合。

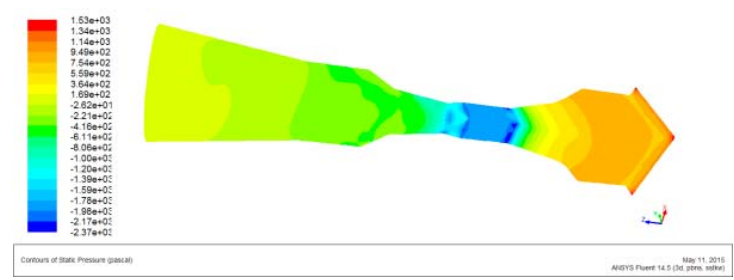

(a) 气流压力场

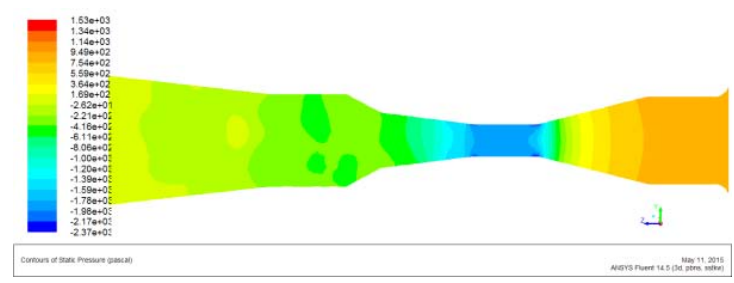

(b) 过轴心水平面压力场

图5 风洞内部气流压力场。

通过ANSYS FLUENT对直流式风洞内部气流进行流 体仿真, 可以深入认识直流式风洞的空气动力学特性, 并 为改进风洞流场品质提供指导。

\section{4. 流固耦合仿真}

根据ANSYS FLUENT计算出风洞内部气流运行时的 压力场分布, 并将该压力载荷分布导入风洞结构静力学模 块STATIC STRUCTURE分析中就可以得到风洞结构受到 内部气流压力产生的应力场及形变分布, 从而实现流体 (风洞内部气流) 和固体 (风洞洞体结构) 的耦合仿真。

\section{1. 网格划分}

图6所示为风洞洞体结构整体和局部边界的四面体网 格划分结果。

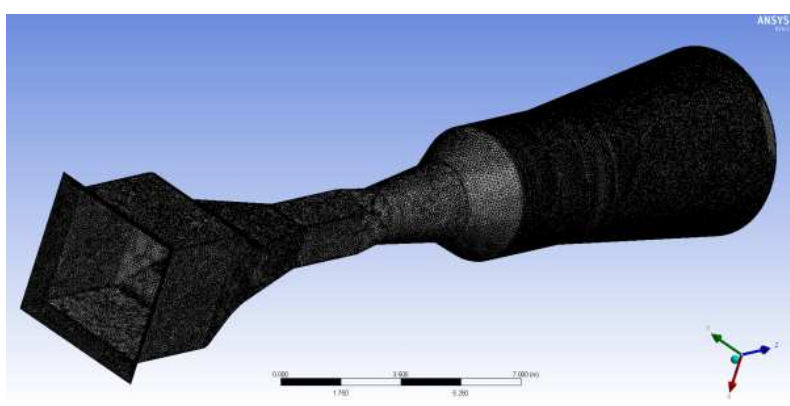

(a) 风洞洞体四面体网格

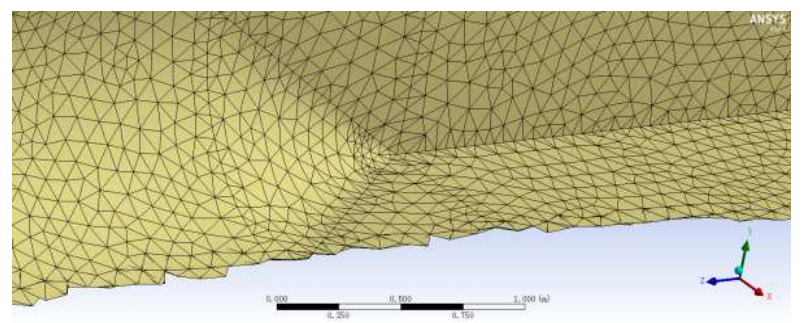

(b) 风洞洞体四面体网格局部

图6 风洞洞体四面体网格。

对风洞洞体结构进行网格划分, 考虑到洞体结构比流 体结构更加复杂, 这里直接采用四面体网格, 通过提高适 当降低网格划分质量来换取网格划分的高效率。同时, 也 能更好的保证网格边界与网格主体的一致性和高质量。风 洞洞体网格划分的单元总数为 49.8 万, 节点数为 99.9 万。

\section{2. 边界条件}

该直流式低速风洞实际上在沿轴线方向有多个支座 支撑, 这里设置试验段底面为固定支撑。原支座位置去掉 了支座模型, 改为固定点支撑, 进行完全约束。其余部段 自由活动 $[10]$ 。

风洞气流载荷来自于内部气流作用于内壁面的压力。 如图7所示, 将ANSYS FLUENT计算的压力场结果映射到 洞体内壁面, 即可得到洞体结构的载荷数据, 并作为 ANSYS STATIC STRUCTURE静力学分析的输入条件, 由 此得到风洞内部气流与风洞洞体结构的耦合计算结果。

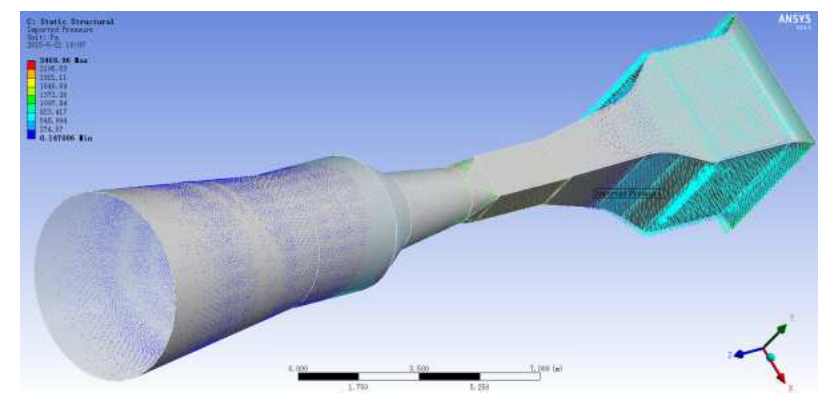

图7 压力场映射载荷。 


\section{3. 仿真结果} 示。

基于ANSYS Workbench的流固耦合仿真结果如图8所

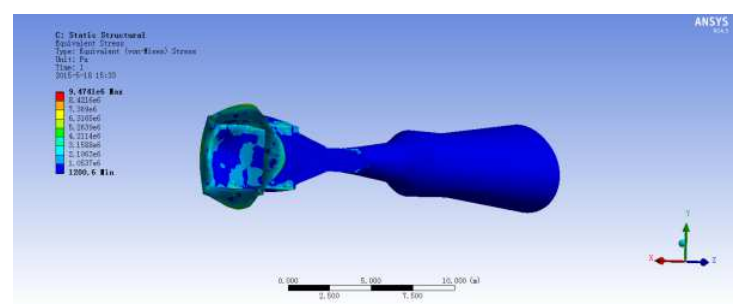

(a) 洞体结构应力分布

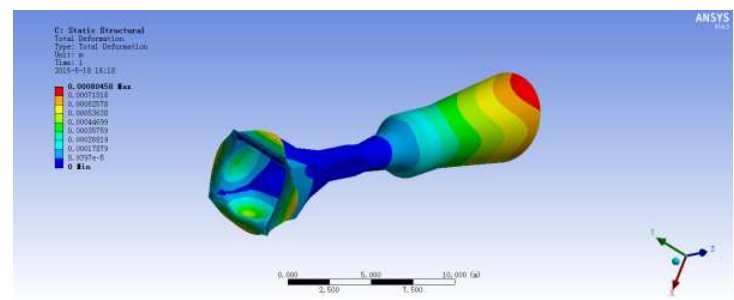

(b) 洞体结构形变

图8 流固耦合仿真结果。

根据数值计算结果, 可以很清楚地认识到风洞结构的 应力分布及形变, 这就为我们后续加强风洞局部结构设计 提供了指导。

\section{5. 结论}

空气动力学研究的实验研究、理论研究和数值计算三 大研究手段相互依赖相互促进。开展基于ANSYS Workbench的直流式低速风洞流固耦合仿真研究, 在开展 实验研究的基础上, 充分利用数值计算的手段对于深入认 识风洞设备的结构强度和气动特性, 认识风洞在各种运行
条件下可能存在的结构、气动、声学等问题有着非常重要 的意义，从而进一步支撑设计人员发现问题并改进风洞气 动结构设计, 改善风洞的空气动力学性能。

\section{参考文献}

[1] 姜新春, 曾劲松, 黄煌. CFD技术在流体机械中的应用与发 展 $[\mathrm{J}]$. 轻工机械, 2014,32(3):108-111。

[2] 陈峰, 王春江, 周岱. 流固耦合理论与算法评述 $[J]$. 空间结 构, 2012,18(4):55-60。

[3] 高飞, 李昕. ANSYS CFX14.0超级学习手册 $[\mathrm{M}]$. 人民邮电 出版社, 2013。

[4] 黄志新, 刘成柱. ANSYS Workbench 14.0超级学习手册[M]. 人民邮电出版社, 2013。

[5] 王丽瑛, 王琪.实用直流式低速风洞的研究 [J].吉林化工学院 学报, 2007,24(2):55-57。

[6] 董国庆, 王仲仁等. FL-9低速增压风洞主题结构有限元分析 与气压试验 $[J]$. 实验流体力学, 2007,21(4):77-82。

[7] 彭威, 王磊, 卢德唐. 局部到整体的六面体网格质量优化算 法[J]. 机械工程学报, 2014,50(3):140-145。

[8] 黄丽丽, 赵国群. 基于栅格法的三维六面体网格质量优化[J]. 中国机械工程，2009,20(21):2603-2607。

[9] 佟晓辉, 王建民, 刘长空等. 环境条件对直流风洞风速测量 结果的影响 $[\mathrm{J}]$. 解放军理工大学学报 (自然科学版), 2014,15(5):494-497。

[10] 解亚军, 郭琦, 肖春生等. NF-6风洞洞体有限元计算与水压 试验 $[J]$. 实验流体力学, 2005,19(2)。 